\title{
Modeling and Performance Analysis of Archimedes Screw Hydro Turbine Using Moving Particle Semi-Implicit Method*
}

\author{
Makoto SHIMOMURA** and Masao TAKANO** \\ ${ }^{* *}$ Graduate School of Environmental Studies, Nagoya University \\ Furo-cho, Chikusa-ku, Nagoya 464-8601, Japan \\ E-mail: s_makoto@nagoya-u.jp
}

\begin{abstract}
This paper presents a numerical model for an open axial-flow hydro turbine using moving particle simulation (MPS) method and discusses the torque generation via the model. An Archimedes-screw hydro turbine, which is a type of open axial-flow hydro turbine, has advantages such as ease of installation, durability, and debris tolerance. However, it has been difficult to analyze dynamically due to a complicated interaction between the open channel flow and the turbine. This paper conducted fundamental experiments such as water flow of a dam moved a flat plate and confirmed the validity of the model. The model reproduced well the rotational movement of the Archimedes-screw hydro turbine in an open channel when no-load conditions. Calculated result of rotational speed and torque distribution showed that the turbine gained the rotational torque by mainly the conversion of a water pressure on the blades due to the water level differences. At the upstream end of the screw, torque loss occurred through a wide range of flow conditions.
\end{abstract}

Key words: MPS Method, Fluid-Structure Interaction, Open Channel Flow, Archimedes-Screw Turbine, Hydro Power Generation

\section{Introduction}

Recently, small-scale hydropower generation (below $2 \mathrm{MW}$ capacity ${ }^{[1]}$ ) has attracted attention, thanks to the wide-spread and stable supply of hydropower at small rivers and irrigation canals ${ }^{[2-5]}$. Micro hydropower generation (below $500 \mathrm{~kW}^{[1]}$ ), especially, is easy to build and set up ${ }^{[6,7]}$. To expand potential sites for electricity generation with economic benefit, the turbine is required to have robust performance with respect to refuse tightness and durability achieved without special debris removal machinery. A type of open axial-flow turbine is known to be practical in terms of ease of installation, durability and tolerance to debris. An Archimedes-screw hydro turbine fits an open, sloped channel and is driven at approximately 10-200 rpm while partially filled with water. It has been reported that the system is $70-80 \%$ turbine efficiency ${ }^{[8]}$, ecofriendly and allowing fish to pass safely ${ }^{[9]}$. While there have been few studies of the turbine itself, the simplified theoretical model in terms of hydrostatics was applied to the turbine ${ }^{[10,11]}$. However, the models are not sufficient to analyze dynamic and local aspects of torque generation because the theory depends strongly on assumption of constant water head and depth, and homogeneous water velocity.

For analysis of the dynamic behavior, the complicated interaction between water flow with a violent free surface and the turbine makes both observation and analysis difficult. In particular, the conventional Eulerian mesh method is not suitable to directly simulate the 
free surface flow, because it is difficult to capture or track the fluid-structure interface. In contrast, the particle method based on a Lagrangian framework is more appropriate for simulating free surface flows as well as the fluid-structure interface ${ }^{[12-15]}$.

Simulations of fluid flows involving a free surface had been carried out with particle methods, such as the smoothed particle hydrodynamics (SPH) ${ }^{[16]}$, the distinct element method (DEM) ${ }^{[17]}$ and the moving particle simulation or semi-implicit (MPS) method ${ }^{[18]}$. The SPH method was mainly designed for simulating compressible flows, the DEM method - for powder and solid flows, and the MPS method - for incompressible flows. In case of incompressible flows, the MPS method has been applied across a broad range of engineering disciplines to compute various flows involving a free surface and fluid-solid interaction ${ }^{[19-21]}$. However, there is a critical problem of pressure fluctuations: the pressure oscillates drastically in both space and time due to numerical instability. In the fluid-structure interaction, such non-physical pressure fluctuations could cause unreal behavior. In this regard, previous studies offered improved methods to suppress instability and enhance performance ${ }^{[22-24]}$.

To investigate the essential factors in evaluating, predicting and improving the performance of the open axial-flow turbine, it is necessary to develop the dynamic analysis model and evaluate the local force to generate the torque by water flow. In this study we have developed a 3D numerical model and applied it to the analysis of interaction between the open channel flows and the turbine, based on the MPS method with an incorporating some earlier improvements. The model was designed to realize accurate, stabilized analysis for dynamic behavior of the Archimedes-screw hydro turbine. It is also applicable to other open-type turbines and advanced multi physics analysis. The validity of the numerical model was quantitatively confirmed by a fundamental experiment in which water flow off the dam-break moved a flat plate. To check the validity of the model, we compared the rotational speed of the Archimedes-screw hydro turbine in an open channel flow without external torque (except for the friction of the bearing) with the simulated results in quasi-steady states. We discuss the torque-generating mechanism of the Archimedes-screw hydro turbine in light of the numerical results of the flow visualization and rotational torque distribution.

\section{Modeling for open channel flow and hydro turbine interaction}

The numerical model for analysis of dynamic behavior of the Archimedes-screw hydro turbine was developed by the MPS method. The PNU-MPS method ${ }^{[24]}$ achieved an increase in speed and improvement of stability at the same time. However, it is not sufficiently accurate and stable for analyzing the interaction between the 3D free surface flow and the complex turbine structure. To counteract these shortcomings, a numerical model was developed based on the PNU-MPS method, in combination with some previous improvements in numerical stability ${ }^{[22-26]}$.

The turbine structure was treated as a rigid body, and the interaction between the fluid and the solid was directly simulated using a passively moving solid model ${ }^{[27]}$. The model was represented by particles with a fixed relative configuration. In the algorithm, both fluid and solid particles were calculated in explicit and pressure terms, regardless of particle type (Fig. 1). The solid particles were hydrodynamically affected by the fluid particles due to momentum transfer. The equation governing solid behavior was solved based on the momentum transfer and modified velocities and coordinates of all solid particles. Consequently, the fluid particles were affected by solid particles through the particle number density variation in subsequent time steps. 


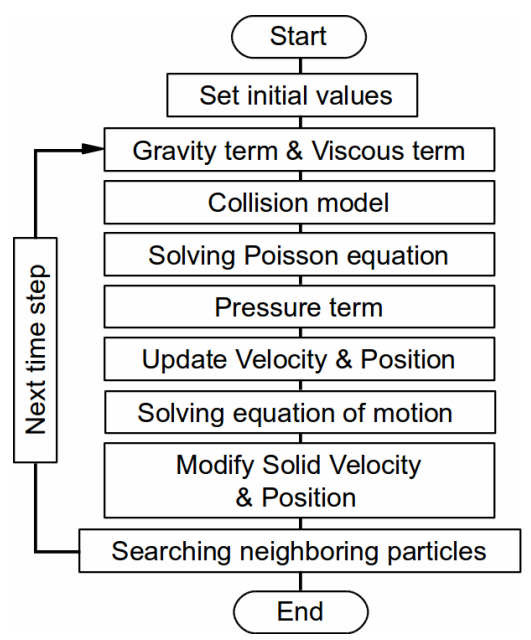

Fig. 1. A schematic description of the MPS-based model algorithm.

\subsection{MPS-based model}

The governing equations for incompressible viscous flows are the continuity equation and the Navier-Stokes equation:

$$
\begin{aligned}
& \nabla \cdot \boldsymbol{u}=0, \\
& \frac{D \boldsymbol{u}}{D t}=-\frac{1}{\rho} \nabla P+v \nabla^{2} \boldsymbol{u}+\boldsymbol{g},
\end{aligned}
$$

where $\boldsymbol{u}$ is the velocity vector, $\mathrm{t}$ - time, $\rho$ - density, $P$ - pressure, $v$ - kinematic viscosity, and $\boldsymbol{g}$ - acceleration due to gravity. The left side of the Navier-Stokes equation denotes Lagrangian differentiation, including a convection term. We took $g=9.81 \mathrm{~m} / \mathrm{s}^{2}, v=1.0 \times 10^{-6}$ $\mathrm{m}^{2} / \mathrm{s}$, and fluid density $\rho_{0}=1000 \mathrm{~kg} / \mathrm{m}^{3}$. The weight function is as follows ${ }^{[24]}$ :

$$
\left.\begin{array}{l}
w_{i j}=\left\{\left(1-\frac{\left|\boldsymbol{r}_{i j}\right|}{r_{e}}\right)^{3}\left(1+\frac{\left|\boldsymbol{r}_{i j}\right|}{r_{e}}\right)^{3}, \quad\left(0 \leq\left|\boldsymbol{r}_{i j}\right|<r_{e}\right)\right. \\
0 \quad, \quad\left(r_{e} \leq\left|\boldsymbol{r}_{i j}\right|\right)
\end{array}\right\}
$$

where $\boldsymbol{r}_{i j}$ is the distance between particles $i$ and $j$, and $r_{e}$ is the effective radius. The sum of the weight functions, the so-called "particle number density" is expressed as the normalization factor by the following equation:

$$
n_{i}=\sum_{j \neq i} w_{i j}
$$

The incompressibility of the fluid is realized by a re-adjustment of the particle number density to the standard $n_{0}$ as constant value determined by the initial conditions. In the $n_{0}$ calculation, fluid particles are usually placed on a lattice. In this case, the structure particles should also be arranged on a lattice. Near the structure wall, particles outside of the wall (so-called "dummy particles") are used in the particle number density calculation. If the method is applied to the structure with complex shape, higher spatial resolution is required. To overcome this kind of problem, there are some correction methods for the particle number density calculation applicable to complex structure walls ${ }^{[28,29]}$. In this study, the 
particle number density was simply calculated using the density fraction of structure particles (Fig. 2). The corrected particle number density $\hat{n}_{i}$ was expressed via the density fraction $\alpha_{i}$ as:

$$
\hat{n}_{i}=n_{0} \alpha_{i},
$$

where $\alpha_{i}$ is calculated as the number density ratio of lattice arrangements to neighboring particles around particle $i$ within the effective radius. In the case of fluid particle calculation, $\alpha_{i}$ was fixed 1.0. The correction enables to set curved surface. The arbitrary particle arrangements, however, should avoid immoderate clustering for stability. The number of neighboring particles around particle $i$ and standard arrangement was defined as $N_{i}$ and $N_{0}$, respectively. Similarly, the corrected number of neighboring particles $\hat{N}_{i}$ was:

$$
\hat{N}_{i}=N_{0} \alpha_{i} .
$$

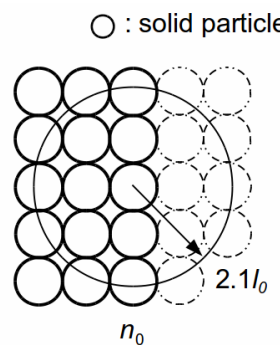

Lattice arrangement

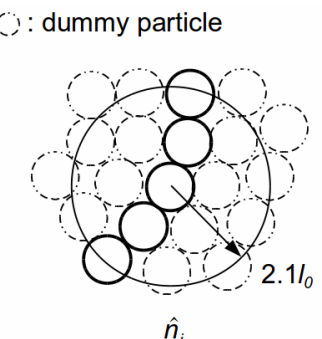

Thin structure

Fig. 2. A schematic diagram of the number density correction.

Next, the above two corrected values were applied to differential operators. The gradient operator was derived from the improved model ${ }^{[23,24]}$ as:

$$
\langle\nabla \varphi\rangle_{i}=\frac{d_{s}}{\hat{n}_{i}} \sum_{j \neq i}\left[\frac{\left(\varphi_{j}+\varphi_{i}\right)}{r_{i j}{ }^{2}} \boldsymbol{r}_{i j} w_{i j}\right],
$$

where $d_{s}$ is the number of dimensions and $\varphi$ is an arbitrary scalar. The differential operator of divergence was derived from the standard MPS model ${ }^{[18]}$ :

$$
\langle\nabla \cdot \varphi\rangle_{i}=\frac{d_{s}}{\hat{n}_{i}} \sum_{j \neq i}\left[\frac{\left(\varphi_{i j} \cdot \boldsymbol{r}_{i j}\right)}{r_{i j}{ }^{2}} w_{i j}\right],
$$

where $\boldsymbol{\varphi}$ is an arbitrary vector. The Laplacian was derived from the 3D higher order model [22]:

$$
\left\langle\nabla^{2} \varphi\right\rangle_{i}=\frac{1}{\hat{n}_{i}} \sum_{j \neq i}\left[\varphi_{i j} \frac{\partial^{2} w_{i j}}{\partial r_{i j}^{2}}\right] .
$$

The governing equation was discretized by the use of these differential operators. The effective radius $r_{e}$ in the gradient and divergence models was $2.1 l_{0}$, in the Laplacian model$3.1 l_{0}$, where $l_{0}$ was the particle diameter.

To improve stability, two kinds of incompressible conditions - so-called "particle number density" and "divergence-free" ${ }^{[23]}$ - were applied. The source term of Poisson's 
equation derives from ${ }^{[24]}$ :

$$
\nabla^{2} P_{i}=(1-\gamma) \frac{\rho_{0}}{\Delta t} \nabla \cdot \boldsymbol{u}_{i}^{*}+\gamma \frac{\rho_{0}}{\Delta t^{2}} \frac{\hat{n}_{i}-n_{i}}{\hat{n}_{i}},
$$

where $\gamma$ is the blending parameter $(0<\gamma<1), \boldsymbol{u}^{*}-$ particle velocity after the explicit calculation. The blending parameter was chosen by two numerical experiments as follows: (a) for a fixed $\gamma$, the relative error rate (RER) of hydrostatic pressure was compared for the MPS method and the analytical solution, and (b) for varying $\gamma$, the time history of pressure on the measuring point was compared by relative standard deviation rate (RSDR) for the calculated results, using the dam-break problem in a 3D rectangular tank (Fig. 3). The RER and RSDR are defined as follows ${ }^{[24]}$ :

$$
\begin{aligned}
& R E R=\frac{\bar{P}_{A}-P_{\text {analyic }}}{P_{\text {analyic }}}, \\
& R S D R=\frac{\sigma_{i}}{\sigma_{\max }},
\end{aligned}
$$

where $\bar{P}_{A}$ is mean pressure at measuring point A (Fig. 3), $P_{\text {analytic }}$ is the analytical hydrostatic pressure $\left(=\rho_{0} g h\right), h-$ water depth, and $\sigma-$ standard deviation. Thus, $\gamma$ is chosen with the goals of both accuracy and variation in both static and dynamic 3D phenomena in Section 3.1.
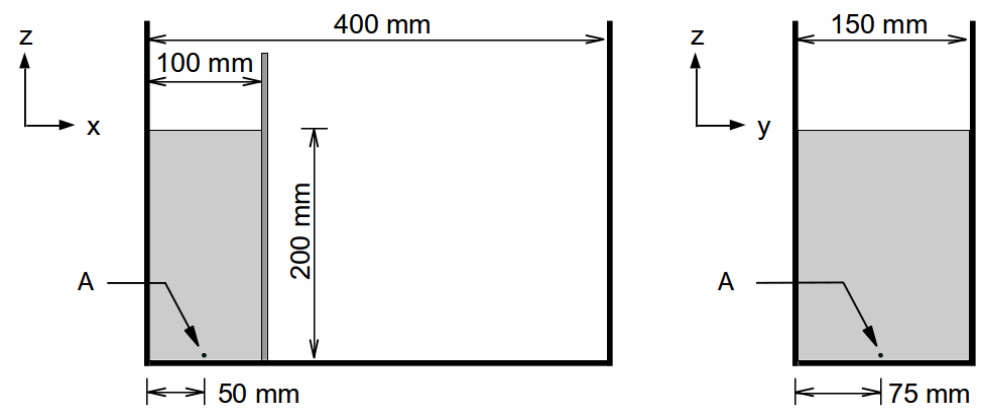

Fig. 3. A schematic diagram of the calculation domain for the 3D numerical experiment.

If the pressure of the misjudged particle is set to zero, this creates a large pressure difference compared to surrounding particles and causes spatial fluctuations in the pressure field ${ }^{[24]}$. Therefore, the free surface particles were determined by the conditions on particle number density $n_{i}$ and the number of neighboring particles $N_{i}$ with two different effective radius, using the corrected standard value $\hat{n}_{i}$ and $\hat{N}_{i}$ following ${ }^{[24]}$ :

$$
n_{i} \leq 0.97 \hat{n}_{i} \cap N_{i} \leq 0.80 \hat{N}_{i},
$$

where the effective radius was $2.1 l_{0}$, and the number of neighboring particles was $3.1 l_{0}$.

When the calculation domain included a boundary, we employed the Neumann condition as the inflow boundary condition and the velocity of the particles on the inflow cross section were set to be constant following ${ }^{[30,31]}$. The Dirichlet condition was employed at the outflow boundary, pressure distribution of particles near the boundary was set to result in a static pressure distribution. 


\subsection{Rigid body motion}

When a solid body rotates with one degree of freedom and friction acts along the axis, the equation of motion is:

$$
I \frac{d \omega}{d t}=T-T_{\text {fric }},
$$

where $I$ is moment of inertia, $\omega$ - angular velocity, $T$ - rotational torque, and $T_{\text {fric }}$ - frictional torque. For a structure rotating around the $x$-axis, the moment of inertia $I$ is:

$$
I=\rho_{\text {solid }} l_{0}^{3} \sum\left[\left(r_{i y}-r_{g y}\right)^{2}+\left(r_{i z}-r_{g z}\right)^{2}\right] .
$$

The rotational torque $T$ is calculated via the velocity of solid particles and the coordinates of the solid particles relative to the center of gravity of the solid body $\boldsymbol{r}_{g}$ :

$$
\begin{aligned}
& T=\rho_{0} l_{0}^{3} \sum \frac{D \boldsymbol{u}_{i}^{\text {solid }}}{D t} \times\left(\boldsymbol{r}_{i}^{\text {solid }}-\boldsymbol{r}_{g}\right) \\
& \boldsymbol{r}_{g}=\frac{1}{N^{\text {solid }}} \sum \boldsymbol{r}_{i}^{\text {solid }}
\end{aligned}
$$

where $N^{\text {solid }}$ is the number of particles in the solid body. The frictional torque $T_{\text {fric }}$ comes from the following simplified function:

$$
T_{\text {fric }}=\frac{D_{s}}{2} \mu F,
$$

where $D_{s}$ is radius of rotation, $\mu$ - coefficient of friction, $F$ - the load acting on the axis. Therefore, the angular velocity vector at the $k$ th step is:

$$
\boldsymbol{\omega}^{k}=\boldsymbol{\omega}^{k-1}+\frac{1}{I} \rho_{0} l_{0}^{3} \sum\left[\left(\boldsymbol{u}_{i}^{\text {solid }}(t+\Delta t)-\boldsymbol{u}_{i}^{\text {solid }}(t)\right) \times\left(\boldsymbol{r}_{i}^{\text {solid }}(t)-\boldsymbol{r}_{g}(t)\right)\right]-\Delta t T_{\text {fric }},
$$

where $\omega_{\mathrm{y}}=\omega_{\mathrm{z}}=0$ when rotating around the $x$-axis. Finally, each solid particle's velocity and coordinates were modified by a quaternion similar to ${ }^{[32]}$.

To apply the thin wall structure composed of solid particles, the solid particles have two pressure parameters and interact with fluid particles located in each surface region. If two fluid particles located on different sides of the wall, they should not interact with each other. To calculate the particle number density near the wall correctly, the wall thickness of the structure is restricted by the effective radius (e.g. if effective radius is $2.1 l_{0}$, the wall particle arrangement needs to more than 3-layer thick). The inner layer of wall particles interacting with the fluid particles should be calculated in the gradient term. The dummy particles are used only in the particle number density calculation.

\section{Validation test}

\subsection{Choosing the blending parameter $\gamma$}

The blending parameter $\gamma$ was determined within the appropriate range of $0.1 \leq \gamma$ via a numerical RER experiment (Fig. 4). RSDR had a minimum point between $0.1 \leq \gamma \leq 0.5$ (Fig. 4). However, there was no definite value which minimized both RER and RSDR. To narrow down the value of $\gamma$, we picked first $\gamma=0.1$ and then $\gamma=0.35$ because both of values 
achieved less than $2 \%$ error of hydrostatic pressure as verification (Fig. 5). Subsequently, comparing the numerical pressure oscillation between $\gamma=0.1$ and $\gamma=0.35$, the oscillation reduction was shown to be greater for $\gamma=0.1$ than $\gamma=0.35$ (Fig. 6). Therefore, the blending parameter $\gamma$ of 0.1 was used for subsequent calculations.

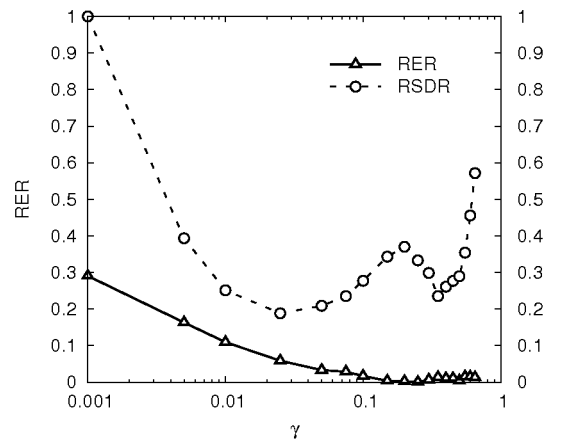

Fig. 4. The calculated results for $\gamma$, where RSDR is normalized by the maximum value at $\gamma=0.001$.

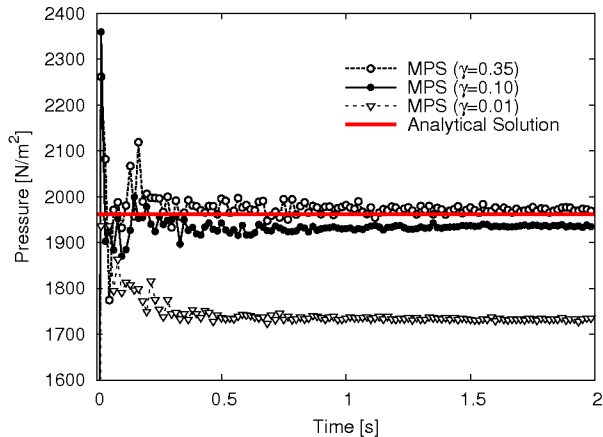

Fig. 5. The time history of analytical and calculated pressures at the measuring point $\mathrm{A}$ (see Fig. 3) with $\gamma=0.35,0.10$ and 0.01 .

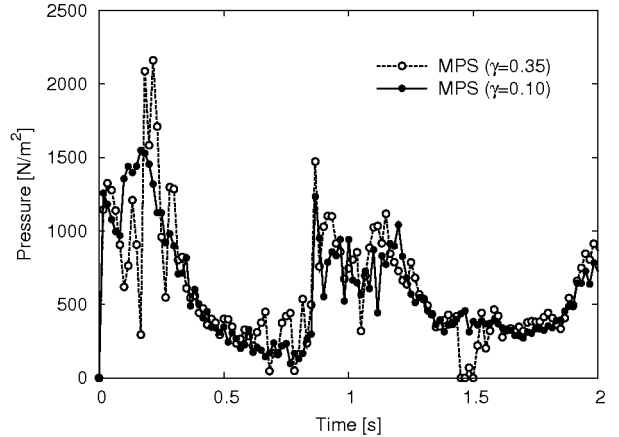

Fig. 6 . The time history of the calculated pressure at the measuring point A (see Fig. 3) for the dam-breaking simulation $(\gamma=0.10$ vs. $\gamma=0.35)$.

\subsection{Dam-breaking flow and a flat plate rotating interaction}

In previous studies of the fluid-structure interaction which included a free surface, there have been some experiments to validate the numerical methods: thrusting away the elastic plate gate by water from a filled tank ${ }^{[13,33]}$, collapsing solid cylinder layers in water ${ }^{[15]}$, and dropping a flat plate in a water tank at an angle ${ }^{[34]}$. This study proposes a new validation experiment suitable for water turbine analysis: interaction between dam-breaking water flow and a flat plate rotating in a rectangular tank (Fig. 7).

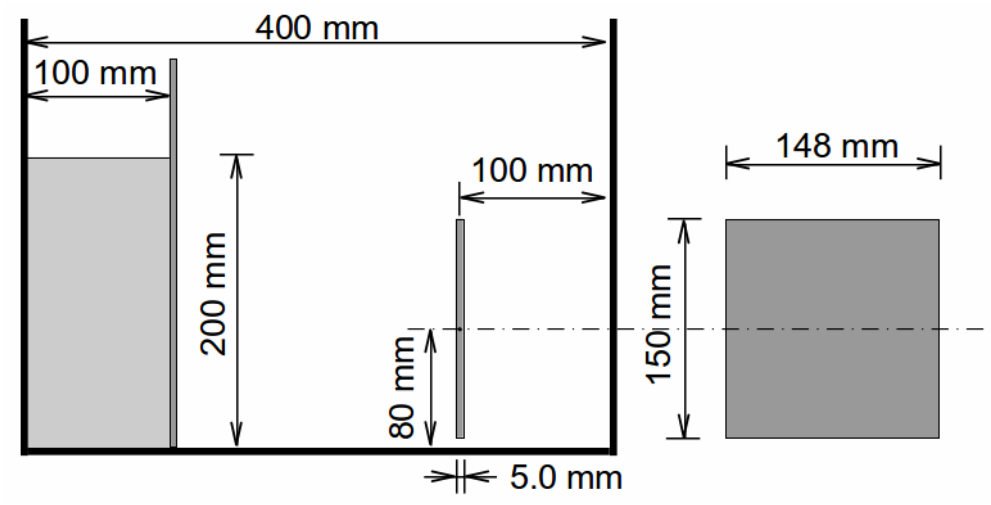

Fig. 7. A schematic diagram of the calculation domain for the validation of fluid-structure interaction. 
To validate fluid-solid coupling in this model, the calculated results have been compared with the experimental ones. In the experiment, the angular velocity of the plate and the shape of water surface were measured for plate density of $2700 \mathrm{~kg} / \mathrm{m}^{3}$, friction coefficient of the bearing of 0.2 , and the speed of the flashboard was $0.78 \mathrm{~m} / \mathrm{s}$. The friction coefficient was calculated by Eqs. (15) and (19), when the plate was subjected solely to frictional torque. The angular velocity was measured via displacement on images captured by 120 frames per second camera, when a freely-rotating plate was slowed down by friction. To evaluate the effect of spatial resolution, the calculated results in the case of two particle diameters $5.0 \times 10^{-3} \mathrm{~m}$ and $2.5 \times 10^{-3} \mathrm{~m}$ were compared to the experimental ones.

The time history of angular velocity of the flat plate is shown in Fig. 8. The error between experimental and calculated results, evaluated by root mean square error (RMSE) and the coefficient of determination $\left(\mathrm{R}^{2}\right)$, is shown in Table 1 . In the calculated results, the time history of the flat plate angular velocity showed good agreement with experimental results. The spatial resolution exhibited little difference between $5.0 \times 10^{-3} \mathrm{~m}$ and $2.5 \times 10^{-3} \mathrm{~m}$ particle diameters, due to the accumulated error of the plate's angular velocity.

The snapshots of the water shape and flat plate motion showed good agreement with the experimental results (Fig. 9). At the first impact between the water and the plate at around $t$ $=0.3 \mathrm{~s}$, there was about $40 \%$ error in the plate rotational speed (Fig. 8). At around $t=0.8 \mathrm{~s}$, there was still large error in the plate rotational speed due to the effect of the first impact. However, the error of plate displacement in the snap shot was relatively small, in this condition, since the plate inertia was more dominant than the torque differential in Eq. (15). In the experimental results at around $t=5.0 \mathrm{~s}$, the water was still sloshing, judging by the shape of the surface. In the calculated results, the water surface was nearly flat due to sloshing oscillations being damped earlier than in the experiment. This is likely because the total energy was not strictly conserved due to the lack of energy conservation in the governing equation. This kind of problem could be overcome through the application of Hamiltonian MPS [35] with a symplectic scheme for a holonomically constrained Hamiltonian system. In this study, to find out the fundamental rotational simulation of Archimedes-screw hydro turbine and develop a simple model, factors such as strict energy conservation, surface tension and turbulence were not considered.

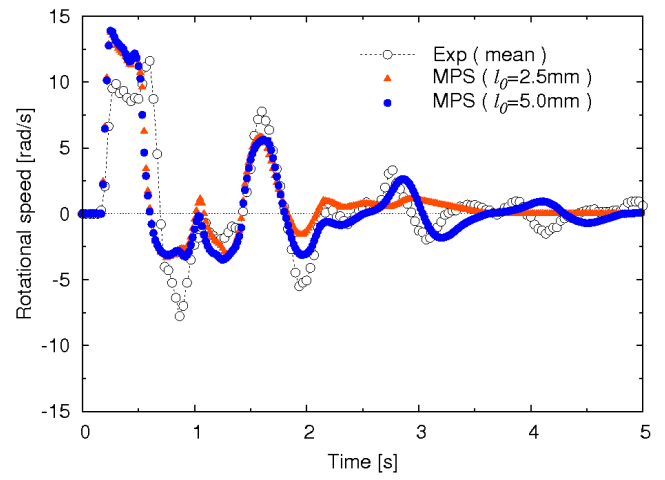

Fig. 8. The time history of the experimental and calculated rotational speeds of the flat plate.

Table 1 Root mean square error (RMSE) and coefficient of determination $\left(\mathrm{R}^{2}\right)$ of the results in Fig. 8.

\begin{tabular}{lll}
\hline Particle size $(\mathrm{m})$ & RMSE $(\mathrm{rad} / \mathrm{s})$ & $\mathrm{R}^{2}$ \\
\hline $5.0 \times 10^{-3}$ & 2.20 & 0.67 \\
$2.5 \times 10^{-3}$ & 2.24 & 0.63 \\
\hline
\end{tabular}




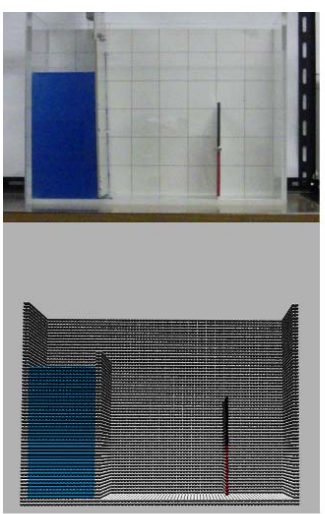

$\mathrm{t}=0.00 \mathrm{~s}$

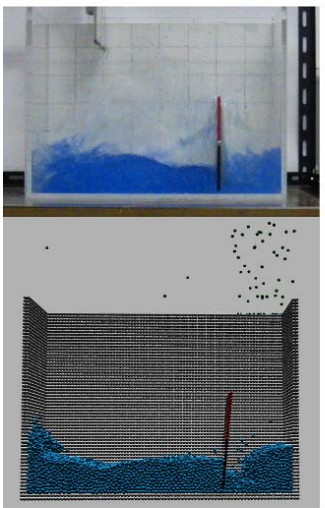

$t=1.00 s$

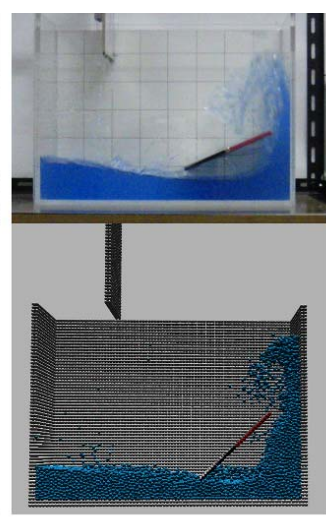

$\mathrm{t}=0.38 \mathrm{~s}$

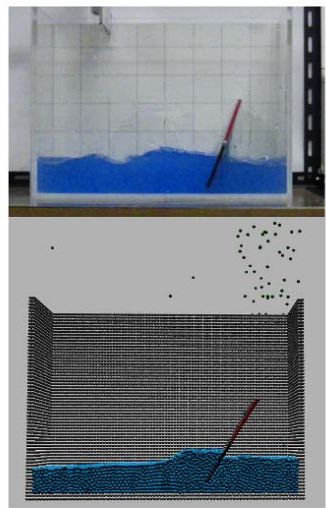

$\mathrm{t}=2.00 \mathrm{~s}$

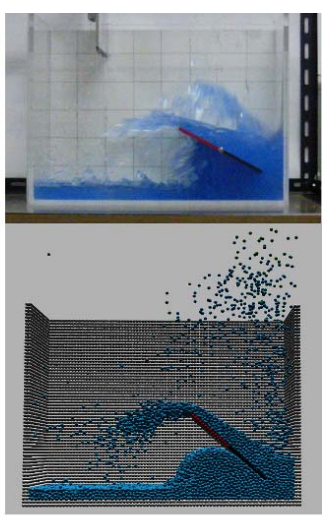

$\mathrm{t}=0.73 \mathrm{~s}$

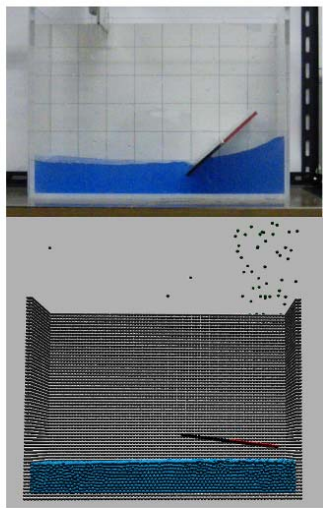

$t=5.00 s$

Fig. 9. A comparison of the MPS calculation and experimental snapshots of the flat plate and dam-breaking water flow interaction. In the calculation, particles escaping the tank were fixed in place.

\subsection{Rotational speed of an Archimedes-screw hydro turbine}

The numerical model was applied to driving a simulation of an Archimedes-screw hydro turbine, involving complicated interactions between open channel flows and a rotational turbine. In the numerical model, the Archimedes-screw hydro turbine was set in the open channel with a U-shaped cross-section (Fig. 10). The geometry of the channel and the turbine yielded the data in Tables 2 and 3, based on the experimental equipment (Fig. 11). The frictional torque was considered to be an external load, and the coefficient of friction was estimated by the governing equation of a solid with angular velocity measured by free-rotating damping with frictional losses. We then compared the calculated results of the quasi-steady rotational speed of the Archimedes-screw hydro turbine with the experimental results. The experimental blade thickness was quite thin compared with the numerical one. If the particle diameter was adjusted within the scale of blade thickness, computational time required would be enormous. To evaluate the effect of blade thickness, turbine rotational speed was compared with various particle diameters in the calculated results. The particle diameter was chosen with regard to spatial resolution. The following particle diameters were selected: $1.0 \times 10^{-2} \mathrm{~m}, 8.0 \times 10^{-3} \mathrm{~m}$ and $6.0 \times 10^{-3} \mathrm{~m}$, with a 20-degree incline and a flow rate of $10.5 \mathrm{~L} / \mathrm{s}$. The calculated time history of the turbine rotational speed showed with the 3 resolutions (Fig. 12). To balance the spatial and time resolutions, this study utilized the particle diameter $l_{0}=8.0 \times 10^{-3} \mathrm{~m}$.

The calculated and experimental results for rotational speed at the quasi-steady state were compared for a total of 25 conditions - five incline angles and five flow rates (Fig. 13). The calculated results showed good agreement with the experimental ones qualitatively, in terms of both variation of the slope angle and the flow rate. In the calculated results, the evaluation of RMSE was $6.4 \mathrm{rpm}$ and tended to overestimate the turbine rotational speed. Nevertheless, the calculated results had a high correlation with 
experimental ones; the coefficient of determination $\mathrm{R}^{2}$ was 0.97 .

In the model, the rotational speed at the quasi-steady state is determined by time integral of Eq. (15) i.e. the ratio of the angular momentum and turbine inertia. The validation problem, therefore, requires simulating the process of torque generation accurately. The comparison results showed good agreement for various conditions, both qualitatively and quantitatively. The developed model showed valid even for complicated interactions between a screw and open channel flows.

Table 2 The characteristics of the open channel in the experimental equipment.

\begin{tabular}{ll}
\hline Parameter & Value \\
\hline Channel length of upstream section $S_{1}$ & $0.32 \mathrm{~m}$ \\
Channel length of slope section $S_{2}$ & $1.44 \mathrm{~m}$ \\
Channel length of downstream section $S_{3}$ & $0.24 \mathrm{~m}$ \\
Channel width $S_{w}$ & $0.397 \mathrm{~m}$ \\
Offset distance between screw and flow channel $S_{d}$ & $0.17 \mathrm{~m}$ \\
\hline
\end{tabular}

Table 3 The characteristics of the Archimedes-screw hydro turbine in the experimental equipment.

\begin{tabular}{ll}
\hline Parameter & Value \\
\hline Number of blades & 4 \\
Blade length $L_{b}$ & $1.00 \mathrm{~m}$ \\
Blade lead $L_{p}$ & $0.40 \mathrm{~m}$ \\
Blade diameter $D_{f}$ & $0.37 \mathrm{~m}$ \\
Blade thickness $l_{t h}$ & $1 \times 10^{-3} \mathrm{~m}$ \\
Axis diameter of screw $D_{a}$ & $0.165 \mathrm{~m}$ \\
Density of screw $\rho_{\text {solid }}$ & $7900 \mathrm{~kg} / \mathrm{m}^{3}$ \\
Mass of screw $M_{s}$ & $52.7 \mathrm{~kg}$ \\
Moment of inertia $\quad I$ & $0.495 \mathrm{kgm}$ \\
Shaft diameter $D_{s}$ & $50.0 \times 10^{-3} \mathrm{~m}$ \\
Coefficient of friction $\mu$ & 0.156 \\
\hline
\end{tabular}

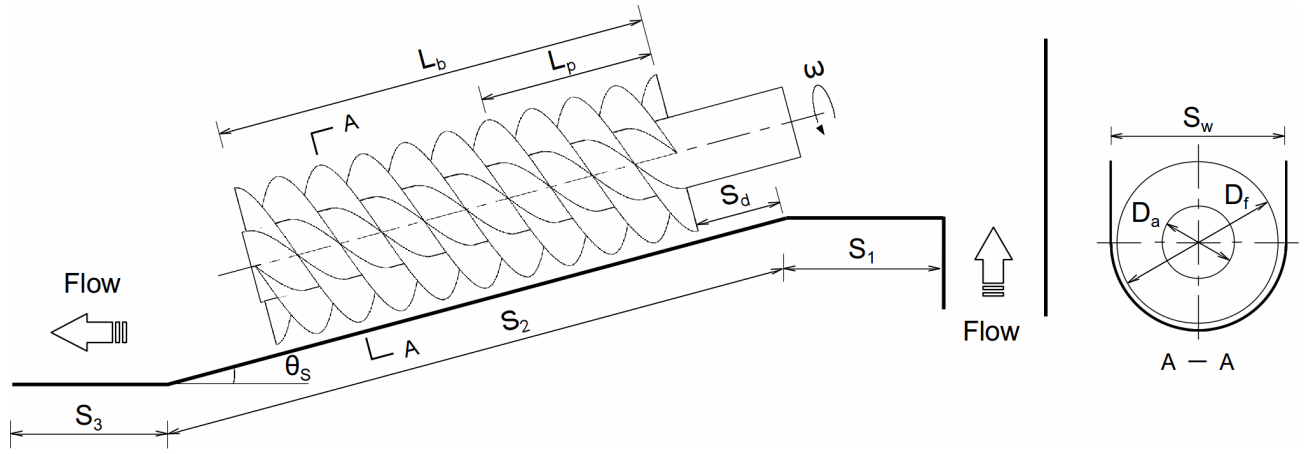

Fig. 10. A schematic diagram of the Archimedes screw and open channel with (left) a cross-section along the flow direction and (right) a cross-section perpendicular to the flow direction. 


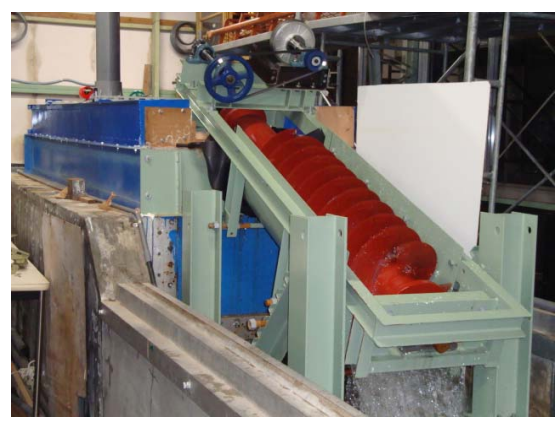

Fig. 11. The experimental equipment of the Archimedes-screw rotating in the open channel.
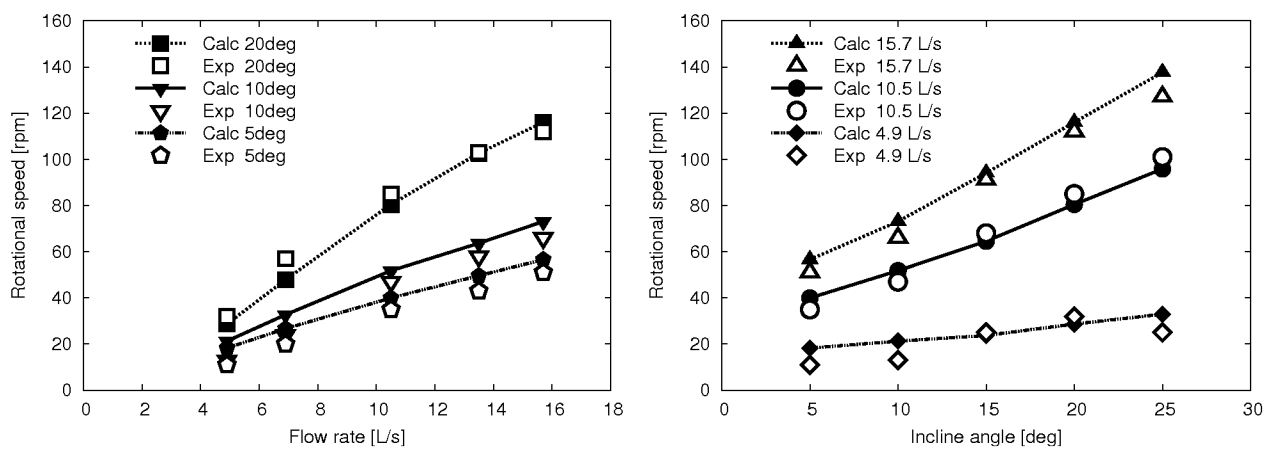

Fig. 13. Experimental and calculated rotational speeds of turbine in the quasi-steady state with (left) different flow rates and (right) different inclines.

\section{Results and Discussion}

\subsection{Visualization of flow and rotational torque distribution}

To investigate the torque-generation mechanism qualitatively, we utilized flow visualization, which enabled us to analyze rotational torque distribution. In particular, we considered the case of the flow rate of $10.5 \mathrm{~L} / \mathrm{s}$ and the incline of $20^{\circ}$. Figure 14 shows the flow appearance and the distribution of the magnitude of the rotational torque on each solid particle using Eq. (17). The contour figures represent the torque averaged over neighboring particles within $2.1 l_{0}$ of each other.

When water was beginning to flow in the channel, if rotational torque became larger than the external load torque, i.e. $T>T_{\text {fric }}$, water rose within the turbine blades located at the upstream end, and relatively large rotational torque appeared on the blade surface (Fig. 14; $t$ $=3.00 \mathrm{~s}$ ). The water remained between neighboring blades as in a bucket, moreover, the volume in each bucket depended on the rotational speed of the turbine and the clearance flow rate. A difference in water levels appeared between the front and the back of the blade surface, rotational torque increased on the lower surface in the axial direction (Fig. 15). In this case, the rotary driving force came mainly from the static pressure difference. The blade surface located at the upstream end caused torque loss due to the static pressure acting from the downstream side, where the water level was higher. When the resulting torque reached a maximum at $t=4.50 \mathrm{~s}$, however, the magnitude of the rotational torque acting on each blade surface was lower than $t=3.00 \mathrm{~s}$. The rotational torque was distributed widely and reached a high value $(t=4.50 \mathrm{~s})$. In the blades located upstream, despite the higher water height within the blades, rotational torque was relatively low due to the small water level difference. When the resulting torque decreased to a negative value, i.e. $T<T_{\text {fric }}$, at $t=6.00$ $\mathrm{s}$, rotational speed reached a maximum due to inertial effects. At the same time, rotational torque decreased, because water height within the blades decreased with increasing rotational speed. With more time, the rotational speed stabilized due to the feedback from 
rotational torque and speed variation, i.e. $T=T_{\text {fric }},(\mathrm{t}=15.00 \mathrm{~s})$. In this regard, the water surface was not horizontal and constant depth within the screw.

As the calculated results of flow and rotational torque distribution show, the static pressure due to the water level deference between the blades indicated the importance of driving and braking forces. These forces are dynamically affected by the water volume in the bucket, which came from both the flow and turbine rotation.
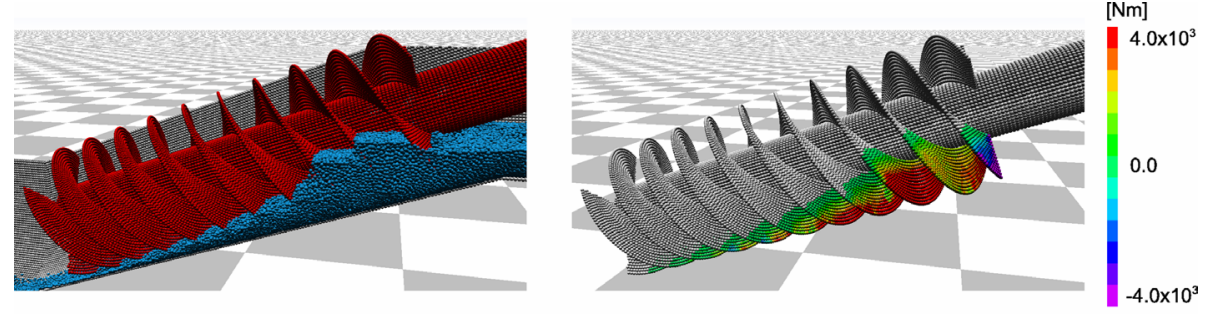

$t=3.00 \mathrm{~s}(\omega=17 \mathrm{rpm})$
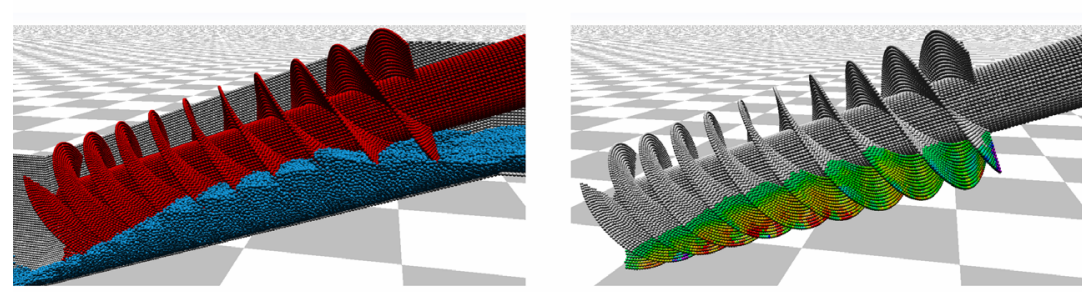

$t=4.50 \mathrm{~s}(\omega=65 \mathrm{rpm})$
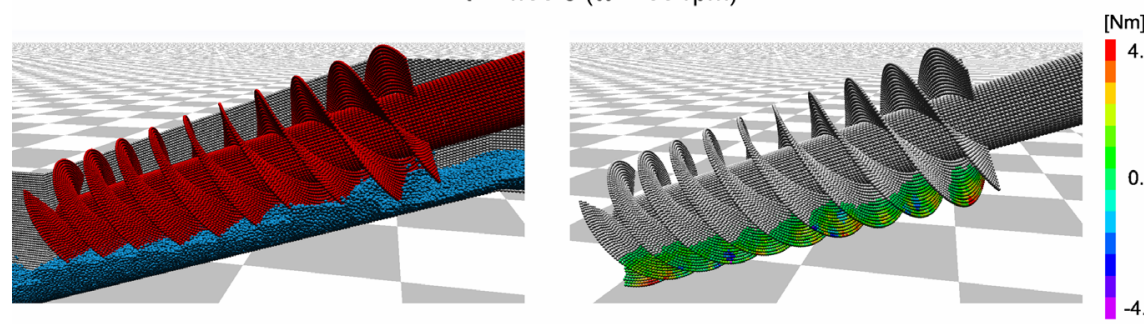
[Nm]

$4.0 \times 10^{3}$

$t=6.00 \mathrm{~s}(\omega=103 \mathrm{rpm})$

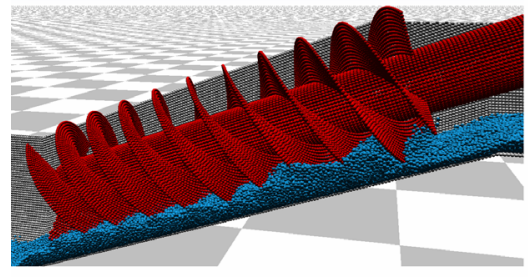

$t=15.00 \mathrm{~s}(\omega=86 \mathrm{rpm})$

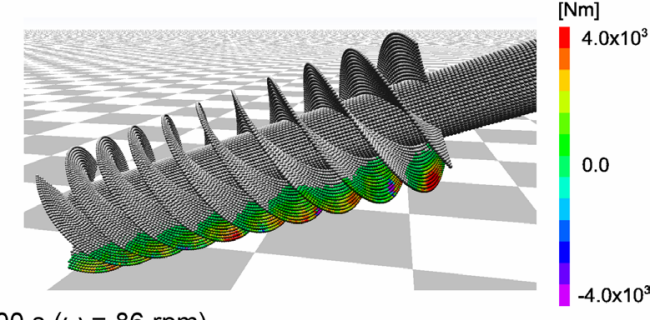

Fig. 14. A flow image at a 20-degree incline with a $10.5 \mathrm{~L} / \mathrm{s}$ flow rate. (Left column: fluid image; right column: the contour of the rotational torque.)
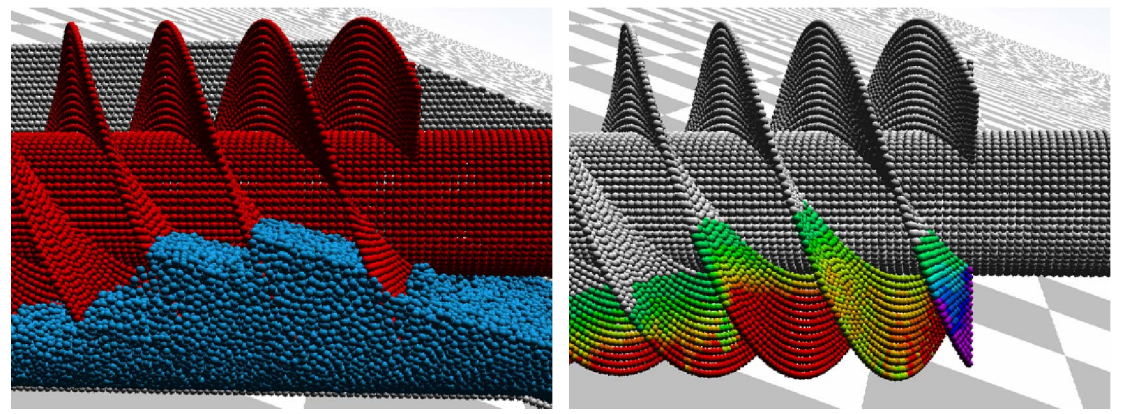

Fig. 15. The water level difference between the blade surfaces in close-up $(t=3.0 \mathrm{~s})$ in the axial direction: (left) flow image and (right) rotational torque. 


\subsection{Analysis of non-dimensional rotational torque distribution}

In order to further investigate the torque-generating mechanism quantitatively, we define non-dimensional torque as the ratio of rotational torque to external load torque at each interval on the local axis (Fig. 16). The distribution of non-dimensional torque $\left(T / T_{\text {fric }}\right)$ averaged over 5.0 seconds in the quasi-steady state is shown with flow rate variation at $\theta_{s}=$ $20^{\circ}$ and with incline angle variation at $Q_{\mathrm{m}}=10.5 \mathrm{~L} / \mathrm{s}$ in Fig. 17 .

For constant incline and varying flow rate, the torque loss arose at the upstream end $\left(x / L_{b}=0.0-0.02\right)$ when the flow rate was large. When the flow rate was $15.7 \mathrm{~L} / \mathrm{s}$, the torque loss was 2.9 and this value corresponds to approximately $5.5 \%$ of the total positive $T / T_{\text {fric }}$. In contrast, when the flow rate was 4.9 and $6.9 \mathrm{~L} / \mathrm{s}$, the non-dimensional torque tended to positive values. For $x / L_{b}=0.0-0.5$, the torque variation was larger when the flow rate was larger. For higher flow rates, the rotation speed increased (see Fig. 13), which decreased the water level difference. The most likely cause was the momentum exchange due to the dynamic pressure between the flows and the turbine blade. Additionally, the water lost its kinetic energy, which likely caused the rotational torque variation (Fig. 17). In this section, however, the rotational torque was approximately $48-50 \%$ of the total for each flow rate (Fig. 18). In the $x / L_{b}=0.5-1.0$ range, the turbine blade experienced relatively constant torque for each flow rate (Fig. 17). Therefore, the blade converted the potential energy due to the incline and turbine shape. In the cross section, the rotational torque was symmetrically distributed around the center of the local $y$-axis for all flow rates.

In the case of constant flow rate, the torque loss arose at the upstream end $\left(x / L_{b}=\right.$ $0.0-0.02)$ for each incline, similar to the constant incline condition. When the incline was $5^{\circ}$, the torque loss was 2.7 ; this value corresponds to approximately $5.2 \%$ of the total positive $T / T_{\text {fric }}$. In particular, when the incline was smaller, the torque loss tended to increase, and rotational torque increased in the downstream section $\left(x / L_{b}=0.5-1.0\right)$. In this section, the percentage of rotational torque was approximately $50-58 \%$ of the total, except for the $5^{\circ}$ incline (Fig. 18). When the incline was $5^{\circ}$, the downstream side generated approximately $70 \%$ of the rotational torque. At this time, when the incline was lower, rotational speed decreased (Fig. 13), and water height within blades rose. In the downstream part of the blade, the water level difference on the blade surfaces increased where the water flowed out, causing relatively large rotational torque by static pressure to the turbine blade. From Fig. 17, in the flow cross section, the rotational torque tended to be distributed asymmetrically with respect to the center of the local $y$-axis when the incline was lower. In particular, when the incline was $5^{\circ}$, the torque loss became large on the left side of the flow for the upstream end. This was also due to static pressure from the downstream side of the blade surface, arising from increased water height. It is necessary to minimize this torque loss to improve performance by improving the turbine configuration.

We have seen that in an Archimedes-screw hydro turbine, the rotary driving force comes from both the static pressure due to the water level difference on blade surface, and dynamic pressure due to momentum exchange with flow. The calculated rotary driving force distribution showed static pressure dominating in the downstream area of the screw, particularly when the incline was below $15^{\circ}$. The water level difference on each blade surface arose from the incline and turbine configuration for a wide variety of conditions.
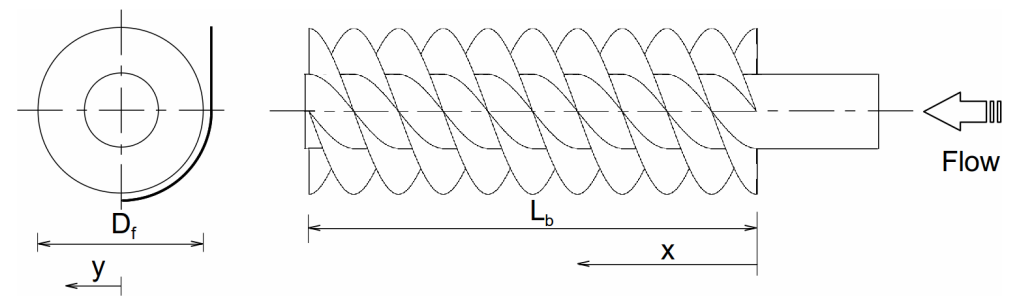

Fig. 16.The local axis of the turbine blade. 

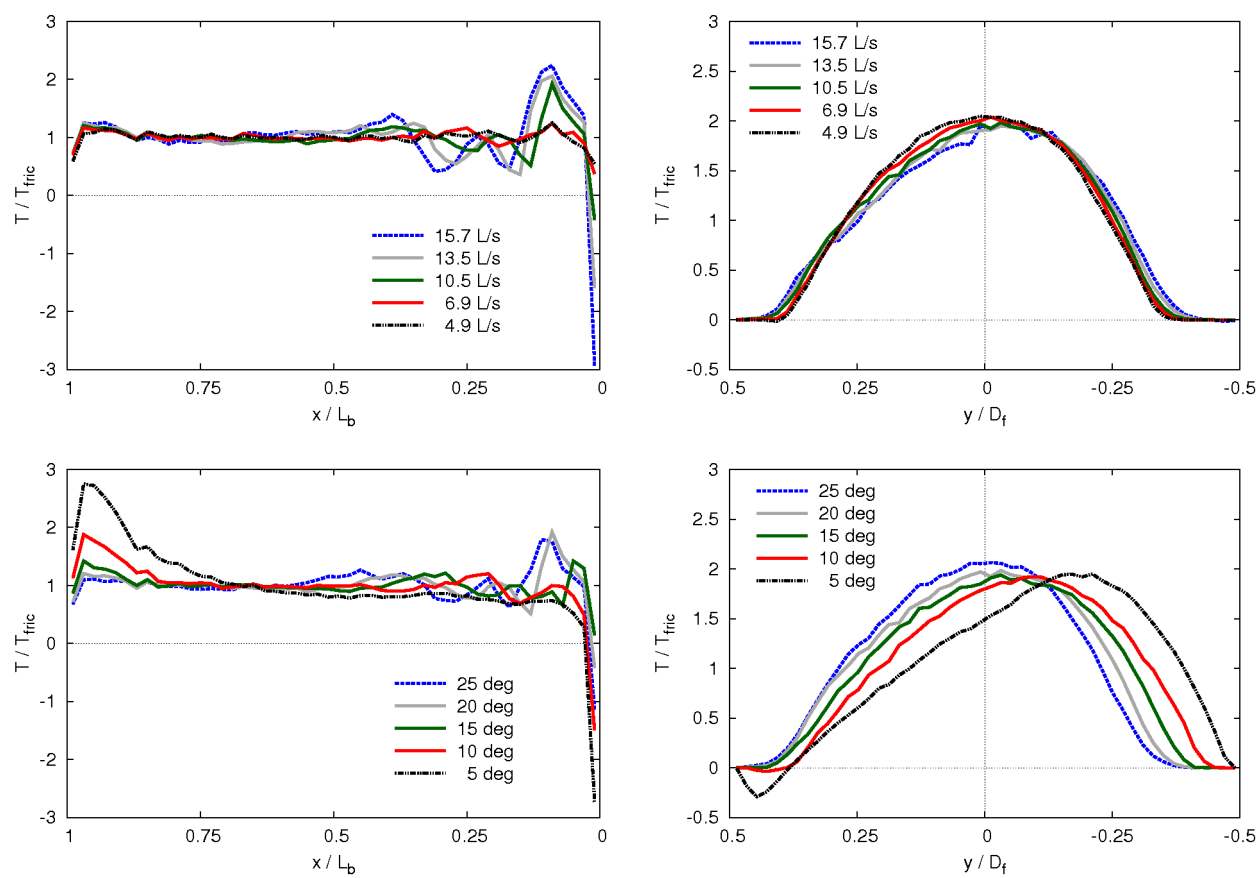

Fig. 17. The distribution of rotational torque at a 20 -degree incline on the upper column (left) local $x$-axis and (right) local $y$-axis with different flow rates, at flow rate $10.5 \mathrm{~L} / \mathrm{s}$ on the lower column.
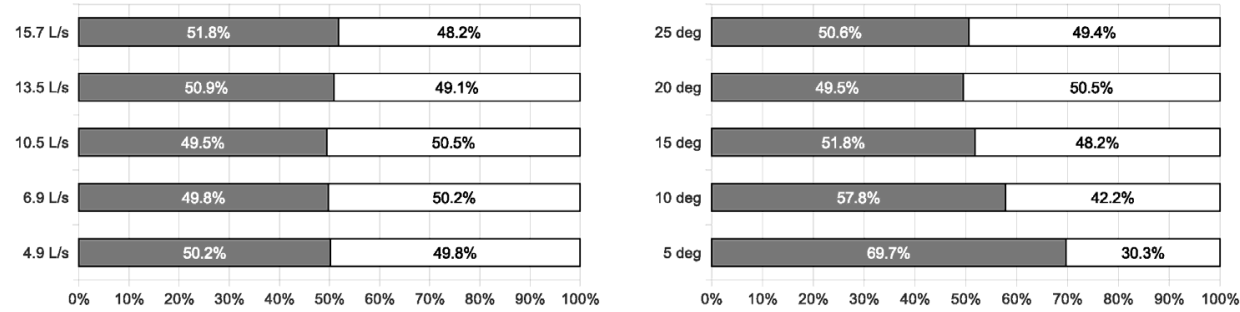

Fig. 18. The percentage of the rotational torque in the downstream area $0.5<x / L_{b} \leq 1.0$ (gray) and the upstream area $0.0 \leq x / L_{b} \leq 0.5$ with (left) different flow rates and (right) different inclines.

\section{Conclusions}

This study has developed a 3D numerical model to analyze the interaction of a hydro turbine and open channel flows. The efficacy of the model was validated by comparing experimental and calculated results of dam-breaking water flow and a flat plate rotating in a rectangular tank, and an Archimedes-screw hydro turbine. The numerical results showed good agreement with experimental results. The model has been shown to be an excellent tool for analysis of open channel flow-turbine interaction.

To investigate the torque-generating mechanism of an Archimedes-screw hydro turbine, the results calculated above were analyzed by employing flow visualization and rotational torque distribution. The fundamental characteristic of the Archimedes-screw hydro turbine is the rotary driving force being generated by both static and dynamic pressures. These forces are generated smoothly by the water level difference on the blade surface and momentum exchange with flows. In the downstream area of the screw, the rotary driving force is dominated by static pressure for inclines below $15^{\circ}$. The water level difference on each blade surface arises from the slope gradient and the turbine configuration for a wide range of conditions. The results show that the flow geometry and turbine rotating are closely related and significantly affect the torque generating mechanism. 


\section{Acknowledgements}

We are grateful to Mr. Hiroyuki Tagami and Prof. Kiyoshi Wada, Gifu national collage of technology, for their valuable support in the execution of the turbine rotating experiments.

\section{References}

[1] Paish, O., Small hydro power: Technology and current status, Renewable and Sustainable Energy Reviews, Vol. 6 (2002), pp. 537-556.

[2] Montes, G.M., Lopez, M.M.S., Gamez, M.C.R. and Ondina, A.M., An overview of renewable energy in Spain: The small hydro-power case, Renewable and Sustainable Energy Reviews, Vol. 9 (2005), pp. 521-534.

[3] Kaldellis, J.K., The contribution of small hydro power stations to the electricity generation in Greece: Technical and economic considerations, Energy Policy, Vol. 35 (2007), pp. 2187-2196.

[4] Ogayar, B. and Vidal, P.G., Cost determination of the electro-mechanical equipment of a small hydro-power plant, Renewable Energy, Vol. 34 (2009), pp. 6-13.

[5] Vicente, S. and Bludszuweit, H., Flexible design of a pico-hydropower system for Laos communities, Renewable Energy, Vol. 44 (2012), pp. 406-413.

[6] Hallett, M., Distributed power in Afghanistan: The Padisaw micro-hydro project, Renewable Energy, Vol. 34 (2009), pp. 2847-2851.

[7] Drinkwaard, W., Kirkels, A. and Romijn, H., A learning-based approach to understanding success in rural electrification: Insights from micro hydro projects in Bolivia, Energy for Sustainable Development, Vol. 14 (2010), pp. 232-237.

[8] Müller, G. and Kauppert, K., Old watermills - Britain's new source of energy?, Proc. ICE Civil Engineering, Vol. 150, No. 4 (2002), pp. 178-186.

[9] Mannpower consulting limited., Fish monitoring and live fish trials - Phase 1, $<$ http://www.mannpower-hydro.co.uk/research.php $>$, (accessed January 2013).

[10] Müller, G. and Senior, J., Simplified theory of Archimedean screws, Journal of Hydraulic Research, Vol. 45, No. 5 (2009), pp. 666-669.

[11] Matsui, J., Internal flow and performance of the spiral water turbine, Turbomachinery, Vol. 38, No. 6 (2010), pp. 358-364 (in Japanese).

[12] Idelsohn, S.R., Oñate, E., Pin, F.D. and Calvo, N., Fluid-structure interaction using the particle finite element method, Computer Methods in Applied Mechanics and Engineering, Vol. 195 (2006), pp. 2100-2123.

[13] Antoci, C., Gallati, M. and Sibilla, S., Numerical simulation of fluid-structure interaction by SPH, Computers and Structures, Vol. 85 (2007), pp. 879-890.

[14] Oñate, E., Idelsohn, S.R., Celigueta, M.A. and Rossi, R., Advances in the particle finite element method for the analysis of fluid-multibody interaction and bed erosion in free surface flows, Computer Methods in Applied Mechanics and Engineering, Vol. 197 (2008) 1777-1800.

[15] Zhang, S., Kuwabara, S., Suzuki, T., Kawano, Y., Morita, K. and Fukuda, K., Simulation of solid-fluid mixture flow using moving particle methods, Journal of Computational Physics, Vol. 228 (2009), pp. 2552-2565.

[16] Gingold, R.A. and Monaghan, J.J., Smoothed particle hydrodynamics - theory and application to non-spherical stars, Monthly Notices of the Royal Astronomical Society, Vol. 181 (1977), pp. 375-389.

[17] Cundall, P.A. and Strack, O.D.L., A discrete numerical model for granular assemblies, Geotechnique, Vol. 29 (1979), pp. 47-65.

[18] Koshizuka, S. and Oka, Y., Moving-particle semi-implicit method for fragmentation of incompressible fluid, Nuclear Science and Engineering, Vol. 123 (1996), pp. 421-434. 
[19] Gotoh, H. and Sakai, T., Key issues in the particle method for computation of wave breaking, Coastal Engineering, Vol. 53 (2006), pp. 171-179.

[20] Shibata, K., Koshizuka, S., Sakai, M. and Tanizawa, K., Lagrangian simulations of ship-wave interactions in rough seas, Ocean Engineering, Vol. 42 (2012), pp. 13-25.

[21] Arai, J., Koshizuka, S. and Murozono, K., Large eddy simulation and a simple wall model for turbulent flow calculation by a particle method, International Journal for Numerical Methods in Fluids (2012) (Published online, DOI: 10.1002/fld.3685).

[22] Khayyer, A. and Gotoh, H., A 3D higher order Laplacian model for enhancement and stabilization of pressure calculation in 3D MPS-based simulations, Applied Ocean Research, Vol. 37 (2012), pp. 120-126.

[23] Tanaka, M. and Masunaga, T., Stabilization and smoothing of pressure in MPS method by quasi-compressibility, Journal of Computational Physics, Vol. 229 (2010), pp. 4279-4290.

[24] Lee, B.H., Park, J.C., Kim, M.H. and Hwang, S.C., Step-by-step improvement of MPS method in simulating violent free-surface motions and impact-loads, Computer Methods in Applied Mechanics and Engineering, Vol. 200 (2011), pp. 1113-1125.

[25] Kondo, M. and Koshizuka, S., Improvement of stability in moving particle semi-implicit method, International Journal for Numerical Methods in Fluids, Vol. 65 (2011), pp. 638-654.

[26] Khayyer, A. and Gotoh, H., Enhancement of stability and accuracy of the moving particle semi-implicit method, Journal of Computational Physics, Vol. 230 (2011), pp. 3093-3118.

[27] Koshizuka, S., Nobe, A. and Oka, Y., Numerical analysis of breaking waves using the moving particle semi-implicit method, International Journal for Numerical Methods in Fluids, Vol. 26 (1998), pp. 751-769.

[28] Chikazawa, Y., Koshizuka, S. and Oka, Y., Numerical analysis of three-dimensional sloshing in an elastic cylindrical tank using moving particle semi-implicit method, Computational Fluid Dynamics Journal, Vol. 9, No. 4 (2001), pp. 376-383.

[29] Harada, T., Koshizuka, S. and Shimazaki, K., A wall boundary computation model by polygons for moving particle semi-implicit method, Proc 8th World Congress on Computational Mechanics (2008), Venice, Italy.

[30] Gotoh, H., Shibahara, T. and Sakai, T., Sub-particle-scale turbulence model for the MPS method - lagrangian flow model for hydraulic engineering, Computational Fluid Dynamics Journal, Vol. 9, No. 4 (2001), pp. 339-347.

[31] Shakibaeinia, A., Asce, S.M. and Jin, Y.C., MPS-based mesh-free particle method for modeling open-channel flows, Journal of Hydraulic Engineering, Vol. 137, No. 11 (2011), pp. 1375-1384.

[32] Johnson, S.M., Williams, J.R. and Cook, B.K., Quaternion-based rigid body rotation integration algorithms for use in particle methods, International Journal for Numerical Methods in Engineering, Vol. 74 (2008), pp. 1303-1313.

[33] Amini, Y., Emdad, H. and Farid, M., A new model to solve fluid-hypo-elastic solid interaction using the smoothed particle hydrodynamics (SPH) method, European Journal of Mechanics B/Fluids, Vol. 30 (2011), pp. 184-194.

[34] Lee, B.H., Park, J.C., Kim, M.H., Jung, S.J., Ryu, M.C. and Kim, Y.S., Numerical simulation of impact loads using a particle method, Ocean Engineering, Vol. 37 (2010), pp. 164-173.

[35] Suzuki, Y., Koshizuka, S. and Oka, Y., Hamiltonian moving-particle semi-implicit (HMPS) method for incompressible fluid flows, Computer Methods in Applied Mechanics and Engineering, Vol. 196 (2007), pp. 2876-2894. 